foetuses exposed to maternal anti-Ro52 autoantibodies. Recent studies investigating other pathogenic autoantibodies (antiinterferon, anti-desmoglein) report that they arise as a result of somatic mutation. The aim of this study was to determine how anti-Ro52 autoantibodies originate.

Methods We traced the evolution of two anti-Ro52 autoantibodies isolated from circulating IgG-switched memory B-cells from a mother of two children with cardiac neonatal lupus. Each antibody was expressed as its immune form or preimmune ancestor by reverting somatic mutations to germline sequence. Antibody reactivity against autoantigens Ro52, Ro60, La and dsDNA were tested by ELISA.

Results Both anti-Ro52 autoantibodies utilised the same heavy and light chain genes (IGHV3-23 and IGLV1-44) but represented distinct clones based on differing complementarity determining region sequences. Anti-Ro52 autoantibodies exhibited a low frequency $(3 \%-4 \%)$ of somatic mutations compared to the average rate of $8 \%$ in healthy switched memory B-cells. In contrast to other pathogenic autoantibodies, the preimmune (germlined) anti-Ro52 autoantibodies showed specific binding to Ro52. However, Ro52 reactivity was higher for the mutated post-immune antibodies compared to their preimmune counterparts demonstrating that autoreactivity was enhanced by affinity maturation.

Conclusions These data demonstrate that Ro52 reactivity is an intrinsic property of the germline antibody repertoire in a mother of children affected by neonatal lupus and indicate defects in central and peripheral tolerance pathways allow propagation of pathogenic autoantibodies.

\section{RESPONSE GENE TO COMPLEMENT-32 PROMOTES PLASMA CELL DIFFERENTIATION AND ENHANCES LUPUS-LIKE CHRONIC GRAFT VERSUS HOST DISEASE}

${ }^{1} \mathrm{H}$ Rus*, ${ }^{1} \mathrm{~A}$ Tatomir, ${ }^{2} \mathrm{~V}$ Nguyen, ${ }^{3} \mathrm{C}$ Cudrici, ${ }^{4} \mathrm{~T}$ Badea, ${ }^{2} \mathrm{~V}$ Rus. ${ }^{1}$ University of Maryland School of Medicine, Neurology, Baltimore, USA; ${ }^{2}$ University of Maryland School of Medicine, Medicine, Baltimore, USA; ${ }^{3}$ National Institute of Health, NIAMS, Bethesda, USA; ${ }^{4}$ National Institute of Health, NEI, Bethesda, USA

\subsection{6/lupus-2017-000215.55}

Background and aims Response Gene to Complement (RGC) -32 plays an important role in cell cycle activation. Our prior studies showed that RGC-32 promotes Th17 differentiation of CD4 T cells. We used wild-type (WT) and RGC-32 knockout (KO) mice to determine whether lack of RGC-32 impairs B cell differentiation and activation and alters autoimmune parameters in the chronic graft versus host disease (cGVHD) model of lupus.

Methods TLR-dependent and $\mathrm{T}$ dependent $\mathrm{B}$ cell differentiation to plasma cells (PC) was induced with LPS and with $\mathrm{CD} 40 \mathrm{mAb}$ plus IL-4. cGVHD was induced with $100 \times 10^{6} \mathrm{Bm} 12$ splenocytes injected into WT or RGC-32 KO recipients.

Results RGC-32 KO B cells failed to differentiate normally to PC as demonstrated by a 2 -fold reduction in PC numbers generated after stimulation and impaired upregulation of $\operatorname{Prdm} 1$ and IRF4 mRNA. RGC-32 transcripts were upregulated in spleen cells from cGVHD mice and protein expression was detected in B cells and germinal centre (GC) cells. RGC-32 $\mathrm{KO}$ hosts displayed an attenuated autoimmune phenotype as demonstrated by decreased production of anti-dsDNA autoantibodies and proliferation of germinal centre $\mathrm{B}$ cells. In addition a decreased number of IgG anti-dsDNA secreting PC and IRF4 and Prdm1 mRNA expression were found

Conclusions These results suggest that expression of RGC-32 in B cells is critical for optimal GC proliferation, PC differentiation and autoantibody production in a murine model of lupus. These data support the idea that RGC-32 blockade has the potential to attenuate autoimmune parameters of cGVHD and possibly reverse abnormalities in the $\mathrm{T}$ and $\mathrm{B}$ cell that contribute to lupus pathogenesis.

\section{MICROMANAGING LUPUS NEPHRITIS: MIR17-92 MODULATES REGULATORY T CELL ACTIVITY BY TARGETING FOXP3 CO-REGULATORS}

${ }^{1} \mathrm{HY}$ Yang, ${ }^{2} \mathrm{CY}$ Wu, ${ }^{2} \mathrm{~L}$ Huang. ${ }^{1}$ Chang-Gung Memorial Hospital- Linko Branch, Nephrology, Taoyuan, Taiwan R.O.C; ${ }^{2}$ Chang-Gung Memorial Hospital- Linko Branch, Paediatrics, Taoyuan, Taiwan R.O.C

\subsection{6/lupus-2017-000215.56}

Background and aims Regulatory $\mathrm{T}$ (Treg) cells play a critical role in maintaining self-tolerance and controlling the magnitude of physiologic immune response. The Treg transcription factor forkhead box P3 (Foxp3) works in concert with other co-regulator molecules including Eos to determine suppressive phenotype of Treg. We identified miR17-92 cluster targeting Eos through bioinformatics approaches.

Methods We generated T-cell-specific miR-17-92 null (mir1792 -/-) mice by mating mir17-92flox/flox mutants to CD4-Cre + transgenic mice. Treg from mir17-92 -/- mice will be isolated, followed by suppression assay to evaluate the role of the miR-17-92 cluster in Treg function. We applied pristane to induce lupus nephropathy in wild type and mir17-92 -/mice. We examined the up-stream promoter region of miR17-92 for binding sites of down-stream mediators of IL-6 signalling, verified by chromatin immunoprecipitation assay.

Results The inflammatory cytokine IL-6 unregulated miR17-92 through HIF-1. MiR17-92 cluster.actively suppressed Eos expression. Knockdown of miR17-92 in Treg enhanced their suppressive activity. Mir17-92 T cell specific deficiency mitigated pristane induced-lupus nephropathy associated with diminished Th17 cells and autoantibody. Moreover, histological analysis revealed a lower mean renal histopathology score and less compliment deposition. Ectopic expression of miR-17 downmodulated the suppression functions of Tregs and provided Treg with partial effector activity via the derepression of cytokine genes.

Conclusions Our studies suggest that miR17-92 modulates Treg cell function by targeting Eos and potentially additional Foxp3 co-regulators, unveiling the future therapeutic potential of microRNA manipulation in lupus nephritis.

\section{A HIGHER FREQUENCIES OF T HELPER 22 CELLS IN PATIENTS WITH NEW ONSET ACTIVE SYSTEMIC LUPUS ERYTHEMATOSUS}

'W Zhong*, 'L Zhao, ' 'Z Jiang, ${ }^{2} Y$ Jiang. ${ }^{1}$ First Hospital of Jilin University, Rheumatology, Changchun, China; ${ }^{2}$ First Hospital of Jilin University, Central Laboratory, Changchun, China

\subsection{6/lupus-2017-000215.57}

Background and aims This study is aimed at elucidating the potential role of Th22 cells in patients with SLE. 